\title{
Gasto y financiamiento en salud: situación y tendencias
}

\author{
Raúl Molina1, Matilde Pinto², Pamela Henderson ${ }^{1}$ y César Vieira ${ }^{1}$
}

RESUMEN El conocimiento del gasto nacional en salud y su financiamiento es esencial para la toma de decisiones respecto a los recursos del sector de la salud y permite evaluar la equidad y la eficiencia con que se asignan y utilizan estos recursos. Las modificaciones en el financiamiento del sector de la salud han sido un componente sustancial de los procesos de reforma del sector en las Américas. El objetivo explícito ha pasado de uno que buscaba exclusivamente la sostenibilidad financiera a otro que busca, simultáneamente, el acceso equitativo a servicios de calidad. La Organización Panamericana de la Salud presenta una propuesta de análisis y diseño de la política de financiamiento del sector cuyo propósito es identificar la combinación de mecanismos de financiamiento que mejor permita lograr los objetivos de sostenibilidad financiera, accesibilidad, equidad y eficiencia. La propuesta consiste en combinar los mecanismos tradicionales de generación de ingresos (gasto público vía impuestos, seguros privados de salud, seguros nacionales de salud y cobro a los usuarios) con mecanismos complementarios de subsidios destinados a compensar a los grupos vulnerables de los efectos de las barreras financieras que reducen su demanda de atención en salud. Las estrategias de financiamiento del sector deben considerar de modo explícito el financiamiento de la atención a las personas y de las intervenciones en el campo de la salud con características de bienes públicos. Para estas últimas se propone el financiamiento público como el más eficiente y equitativo.

El análisis de las fuentes de financiamiento y del gasto del sector de la salud es un tema relevante por su impacto no solo en la sostenibilidad financiera de los sistemas de salud, sino también en el acceso que efectivamente la población puede lograr. El análisis de la composición de las fuen-

\footnotetext{
1 Programa de Políticas Públicas y Salud, División de Salud y Desarrollo Humano, Organización Panamericana de la Salud/Organización Mundial de la Salud (OPS/OMS). Toda la correspondencia debe ser enviada a Raúl Molina a la siguiente dirección postal: Programa de Políticas Públicas y Salud, PAHO/OPS, 525 Twenty-third Street, NW, Washington, D.C. 20037, Estados Unidos de América. Correo electrónico: molinara @paho.org

2 Programa de Organización y Administración de Sistemas y Servicios de Salud, División de Desarrollo de Sistemas y Servicios de Salud, OPS/OMS. Washington, D.C.
}

tes de financiamiento y su comparación con el acceso diferencial a los servicios de salud por los distintos grupos de población permite extraer conclusiones sobre el grado de equidad financiera del sistema. Los datos disponibles en la Región de las Américas sugieren que todavía queda mucho por hacer en términos de equidad, tanto en el financiamiento como en el acceso a los servicios de salud. ${ }^{3}$

El conocimiento del gasto nacional en salud (GNS) y su financiamiento es esencial para la toma de decisiones

\footnotetext{
3 Pan American Health Organization/United Nations Development Programme/World Bank. Health systems inequalities and poverty in Latin America and the Caribbean. Documentos presentados en la reunión del 5-6 de octubre de 1999, Washington, D.C.
}

acerca de la asignación de los recursos del sector de la salud, permite evaluar la equidad y eficiencia con que se asignan y utilizan estos recursos y es clave para comparar los esfuerzos que hacen las sociedades y los gobiernos de los países para enfrentar los problemas de salud que afectan a sus poblaciones. En este sentido, constituye un instrumento técnico que permite informar el diálogo que se da en distintos niveles de la sociedad: entre la sociedad civil, el Estado y el sector privado con respecto a las prioridades y a las políticas de salud; entre las autoridades de salud y del área económica sobre la asignación de recursos al sector, y entre las entidades encargadas de la regulación, gestión, financiamiento y 


\section{La descentralización financiera en Chile en la década de los noventa}

\author{
César Oyarzo M. ${ }^{1}$
}

En diciembre de 1999 se promulgó un nuevo cuerpo legal que reforma las funciones del Fondo Nacional de Salud (FONASA). En esta ley se vuelca la experiencia de un proceso que se inició a comienzos de los años noventa y que tuvo su fase de mayor desarrollo a partir de 1994. En este artículo se resumen las principales características de este proceso, poniendo énfasis tanto en los logros como en los fracasos. Aunque en este sentido se trata de una evaluación crítica de una experiencia en desarrollo, no puede ser una revisión del impacto sobre la salud, ya que este tipo de transformaciones se producen a largo plazo. El artículo se centra pues en los aspectos técnicos de la reforma, dejando para la última sección algunos aspectos de la economía política del cambio.

\section{La propuesta del primer Gobierno de la Concertación}

El modelo de salud chileno sufrió profundas transformaciones durante el régimen militar. A comienzos de la década de los ochenta se produjeron una serie de reformas del sistema de salud (RS), entre las que cabe destacar: 1) la creación del Fondo Nacional de Salud (FONASA), encargado de recaudar los ingresos provenientes del presupuesto nacional y de las cotizaciones obligatorias para la salud, y de administrar el conjunto de las transferencias financieras del sector; 2) el reforzamiento del papel del Ministerio de Salud como agente de decisión de las políticas y como entidad normativa, supervisora y evaluadora; 3) la descentralización del Servicio Nacional de Salud (SNS) en 26 servicios autónomos regionales, que dio origen al Sistema Nacional de Servicios de Salud (SNSS); 4) el traspaso a las municipalidades de la responsabilidad por los servicios de atención primaria y de su infraestructura, cambio iniciado en 1981 y finalizado en 1987, y 5) la posibilidad para los trabajadores de elegir entre adherirse al sistema público de salud (FONASA) o a compañías de seguro de salud privadas: las Instituciones de Salud Previsional (ISAPRE), que comenzaron a operar en 1981.

Desde entonces, en materia de aseguramiento coexisten dos tipos de seguros: uno público (FONASA), de tipo solidario, que cubre a los indigentes y a los cotizantes de menores rentas, y otro privado. El seguro público, siguiendo la tradición de la seguridad social, ofrece un plan único de salud de

\footnotetext{
${ }^{1}$ Fundación Salud y Futuro, Santiago, Chile. Integra Médica, c/Coyancura 2241, Of. 73, Providencia, Santiago, Chile. Correo electrónico: coyarzo@integramedica.cl
}

producción y los usuarios de los sistemas de salud.

Durante la última década ha habido un considerable progreso en la estimación del GNS y su financiamiento en los países de América Latina y el Caribe. Tradicionalmente, el gasto en salud de los gobiernos nacionales y de las instituciones públicas de seguridad social se ha conocido gracias a las informaciones que los países recolectan a través del Sistema de Cuentas Nacionales de Naciones Unidas y comunican al Fondo Monetario Internacional y a la Organización Internacional del Trabajo. En 1993 el Banco Mundial (1) publicó los primeros datos sobre el gasto privado en salud en la Región. Un año después, la Organización Panamericana de la Salud (OPS) (2) publicó sus estimaciones del gasto privado, que indicaron que el promedio regional era mucho más alto de lo que hasta entonces se pensaba. Posteriormente, otras publicaciones (3-5) confirmaron esta tendencia. Desde hace dos años se ha iniciado en la Región un proceso de estimación de Cuentas $\mathrm{Na}$ cionales de Salud (6) con una metodología que permite obtener datos más detallados y más comparables internacionalmente. Este proceso se está realizando gracias al esfuerzo de los países y a la cooperación del Banco Mundial, del Banco Interamericano de Desarrollo, de la Agencia de los Estados Unidos para el Desarrollo Internacional (USAID), de la OPS/OMS y de la Universidad de Harvard.

Este artículo analiza el GNS y su financiamiento en América Latina y el Caribe. Inicialmente se describe y analiza la evolución del GNS total y per cápita, su relación con el producto interno bruto (PIB) de los países y su distribución entre los sectores público y privado. Luego se analiza la participación de las principales fuentes públicas y privadas en el financiamiento del GNS, así como la asignación del gasto a diferentes servicios e insumos y al beneficio de diferentes grupos sociales. Finalmente, se consideran las tendencias de cambio del GNS y de su financiamiento a consecuencia de las modificaciones que ha experimentado el sector de la salud, debido tanto a su 
propia evolución como a los procesos de reforma, y se hace una propuesta de análisis de las fuentes de financiamiento y de diseño de la política de financiamiento del sector en función de los objetivos de la reforma.

Las estimaciones de los promedios regionales que se presentan en este artículo excluyen a los Estados Unidos de América y a Canadá para evitar el sesgo que supone la alta participación de ambas economías en el PIB regional y la alta proporción del PIB que se destina a financiar la salud en estos dos países. De hecho, a mediados de los años noventa, el GNS de los Estados Unidos y Canadá representaba 91\% del gasto en la Región de las Américas y tan solo los Estados Unidos realizaban $86 \%$ de este gasto.

\section{GASTO EN SALUD EN LAS AMÉRICAS}

El gasto en servicios de salud en las Américas durante la última década presenta ciertas características que son producto de las transformaciones del modelo económico, de la redefinición del papel del Estado en la economía, del fortalecimiento del papel de la sociedad civil en el campo de la salud y de las modalidades con que la reforma del sector se ha aplicado a las políticas de financiamiento de los servicios de salud.

La primera de estas características tiene que ver con una gran heterogeneidad del GNS per cápita, no solo por los elevados valores observados en los Estados Unidos y Canadá en relación con el resto de la Región, sino también entre los demás países de la Región (4). Así, por ejemplo, el GNS medio per cápita para toda la Región de las Américas es de US\$392, pero mientras en las Islas Caimán es más de tres veces superior al promedio regional, en Haití representa tan solo $6,1 \%$ de dicho promedio (figura 1). Destaca que ocho países de la Región destinan menos de US\$ 100 per cápita anuales a la salud.

La proporción del GNS en relación con el PIB se suele utilizar como un indicador de la prioridad que la sociedad asigna a la atención de la salud de su población. No obstante, el resultado carácter implícito para todos sus beneficiarios, con dos modalidades de atención (institucional y de libre elección). Por su parte, las ISAPRE son seguros privados, basados también en una cotización obligatoria, pero donde existen múltiples planes de aseguramiento cuyas primas se ajustan a los riesgos y cobertura pactada entre la ISAPRE y el asegurado. Así, cada plan tiene un precio según el sexo, edad y cobertura.

La coexistencia de un sistema de seguros privados con un sistema de seguro público, de corte solidario, bajo un esquema de cotización obligatoria establecida como un porcentaje del ingreso, genera una segmentación clara entre diferentes grupos por niveles de riesgo y niveles socioeconómicos. El sistema privado atiende en la actualidad a $26 \%$ de la población y recibe $69 \%$ de los recursos recaudados por cotizaciones, mientras que el sistema público atiende a $64 \%$ de la población y obtiene $31 \%$ del total de cotizaciones del sistema. Otro elemento que apunta en la misma dirección es el siguiente: en 1994 la cotización anual media del sistema ISAPRE fue de 241300 pesos chilenos, mientras que en el sistema público fue de 72459 pesos chilenos, es decir, menos de un tercio de la cotización anual promedio del sistema privado.

El proceso de descentralización del sistema público, introducido en la década de los ochenta, no tuvo los resultados esperados. El Ministerio de Salud careció de una organización que le permitiera desarrollar su tarea central de definir políticas y supervisar su cumplimiento. A su vez, los Servicios de Salud Regionales enfrentaban problemas importantes en la ejecución de sus funciones debido, en parte, a la existencia de importantes rigideces laborales que dificultaban una efectiva descentralización y a la falta de especialización de sus administradores en materias de gestión. Ello llevó a que, en la práctica, los Servicios de Salud continuaran dependiendo del Ministerio de Salud, aun en aquellos aspectos en los que poseen plena autonomía. En el caso del FONASA, las facultades otorgadas por la ley no fueron asumidas, ya que el ministerio continuó administrando los fondos destinados a los hospitales públicos. De esta manera, la incipiente descentralización funcional no funcionó. Por su parte, la experiencia de traspaso de la atención primaria a las municipalidades tampoco resultó según lo esperado. Si bien en algunos municipios se fortaleció la atención, en la mayoría de ellos la desintegración del sistema generó un ambiente de insatisfacción entre los médicos que contribuyó a una alta rotación de profesionales. En resumen, el proceso de descentralización fue incompleto e imperfecto. El mayor avance relativo tuvo lugar en el campo de la descentralización geográfica, pero en materia de descentralización funcional los avances fueron escasos.

El primer Gobierno de la Concertación definió como principios orientadores de su política de salud: mejorar el acceso a la salud de la población y, en particular, de los más pobres; iniciar la rehabilitación de la red hospitalaria pública; fortalecer la prevención y promoción de la salud; mejorar la gestión e iniciar el desarrollo institucional del sector público de la salud.

Un primer paso para mejorar la equidad en el acceso a la atención de la salud de los grupos más pobres y vulnerables fue asignar recursos adicionales al sector público, cuyos principales beneficiarios eran estos grupos de la población. Gracias a una reforma tributaria que elevó los impuestos generales, se pudo incrementar el gasto público en salud en un 33\% real en el período 1990-1992. 
FIGURA 1. Gasto per cápita en salud (público y privado), 1997-1998

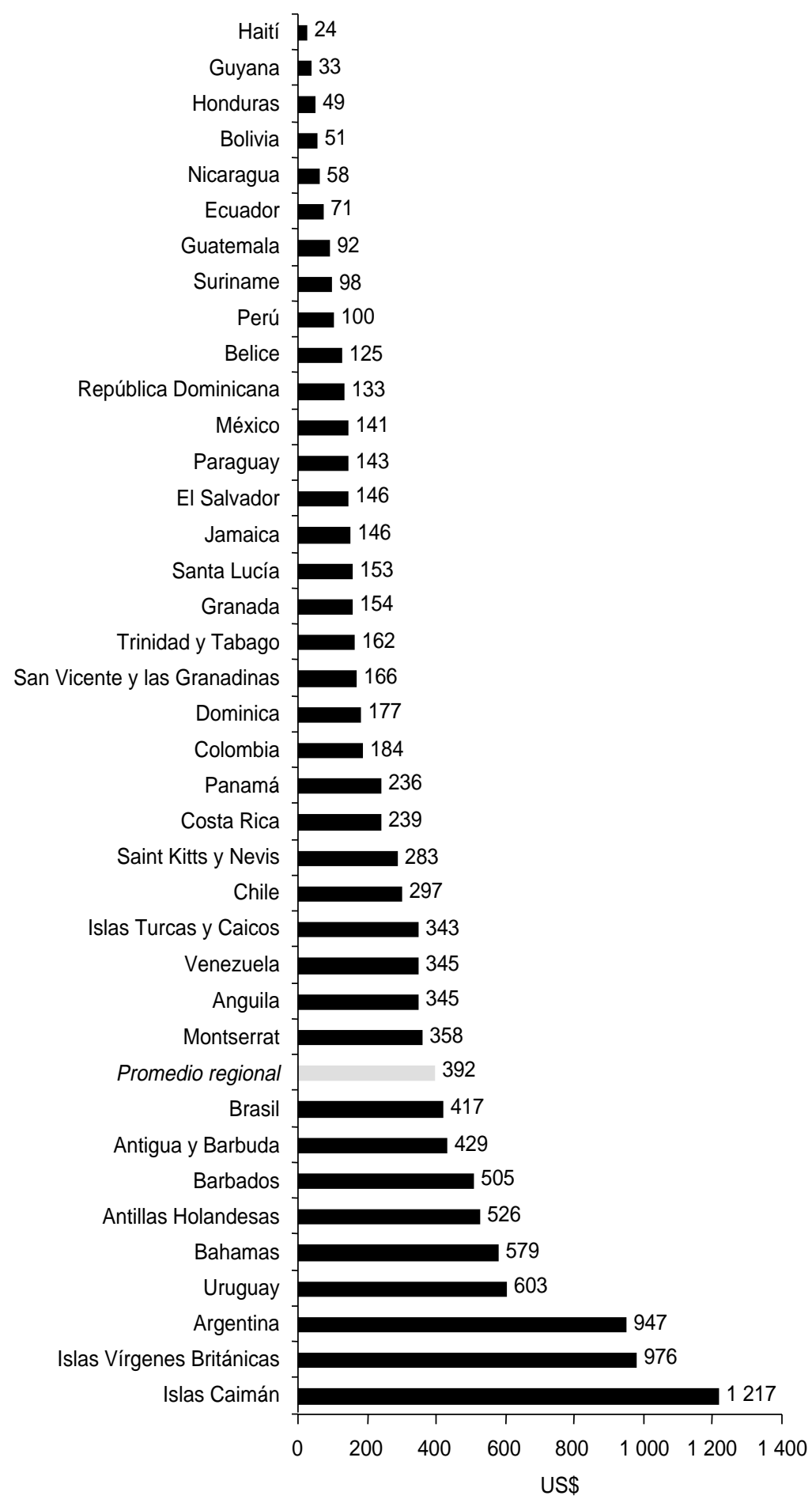

final depende del destino de los recursos y de la calidad del gasto en términos de equidad, efectividad y eficiencia. Por esta razón, se considera que en la Región de las Américas el GNS es elevado en relación con los resultados que se obtienen. El GNS medio regional como proporción del PIB fue de $7,7 \%$ a finales de la década (7) y mostraba una tendencia creciente; sin embargo, en 15 países de la Región no alcanzaba 5\% del PIB (figura 2).
En segundo lugar, y en apoyo de la tesis de que es posible mejorar la efectividad y la eficiencia del gasto, se observa que el GNS per cápita en las Américas es, en términos absolutos, más elevado que en otras regiones del 
FIGURA 2. Gasto total en salud (público y privado) como porcentaje del producto interno bruto (PIB), 1997-1998

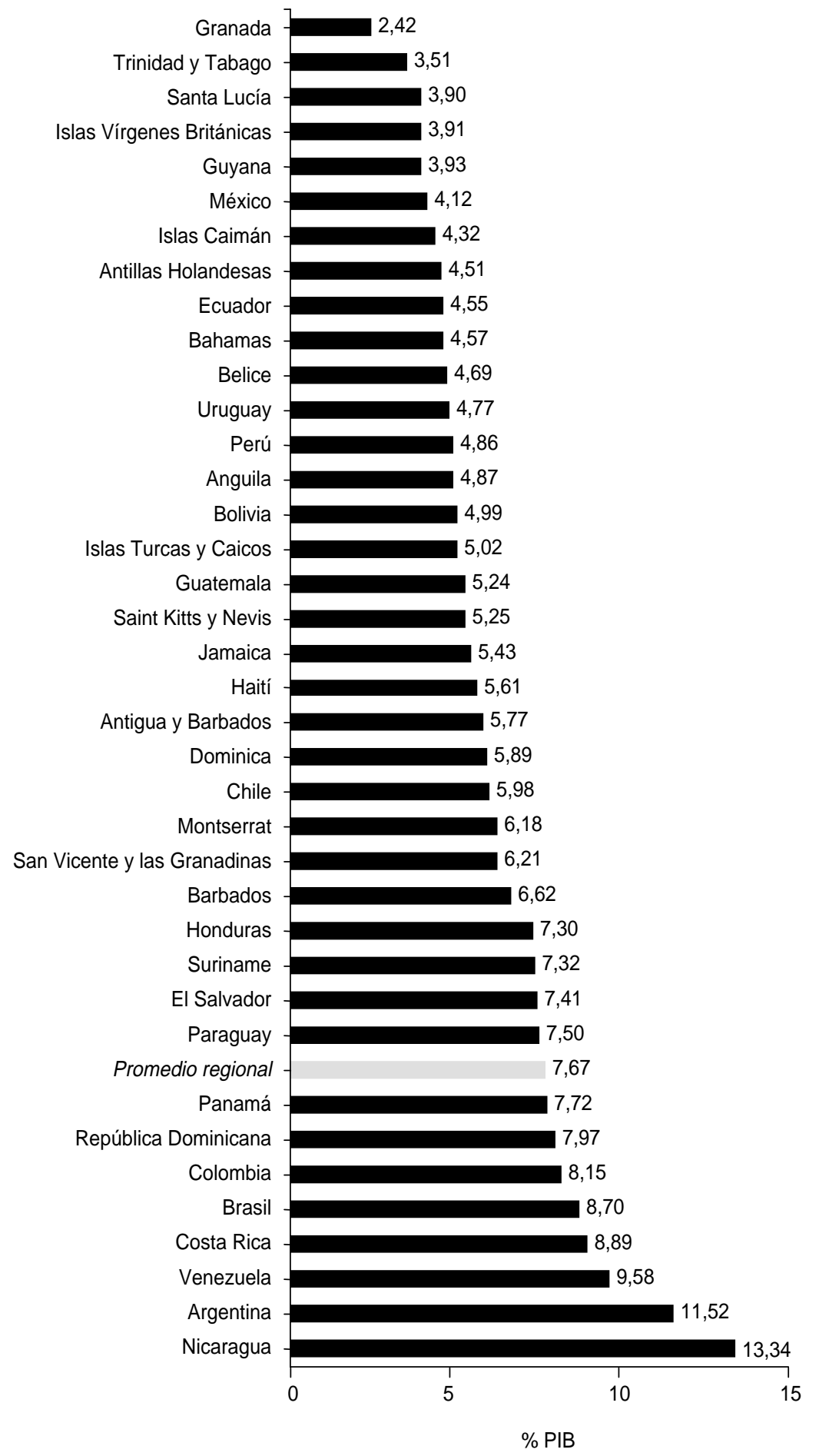

mundo, como Asia o África. En términos relativos, este gasto es muy cercano al de los países industrializados (cuadro 1). El promedio regional de $6,1 \%$ del PIB destinado al gasto en salud refleja el hecho de que un grupo importante de países de la Región, en especial aquellos con una baja participación del sector público y con esquemas de financiamiento plural, destinan menos de $5 \%$ del PIB a la salud.
En tercer lugar, se observa una limitada participación del sector público en el GNS, al contrario de lo que pasa en otras regiones del mundo. Esta situación es preocupante, ya que el gasto público está siendo reemplazado por 
De forma paralela, se plantearon las primeras orientaciones de la RS para asegurar la eficiencia y equidad del sistema. La reforma en el subsector público incluyó: a) la readecuación del sistema de atención de salud para acondicionarlo a las necesidades surgidas del nuevo perfil epidemiológico del país; b) el perfeccionamiento del modelo de gestión del sector público para fortalecer sus capacidades de regular, financiar, ejecutar y supervisar, para lo cual se planteó la reorganización del Ministerio de Salud y de los servicios de salud, con el fin de producir una mayor descentralización, y c) la reforma de los mecanismos de asignación de recursos financieros al sector público, con el propósito de adecuar los incentivos a las metas de salud.

Durante el período 1990-1994, el gasto público en salud experimentó un incremento real de $65 \%$, con un aumento del aporte fiscal del orden de $100 \%$. La mayor parte del incremento del gasto fue asignada a mejorar la deteriorada infraestructura de los establecimientos públicos. Esta inversión se materializó tanto en obras físicas como en equipamiento. El segundo componente del gasto que aumentó fue el gasto en remuneraciones. Este incremento fue el resultado tanto de expansiones en la dotación de funcionarios como de reajustes de los salarios medios. Adicionalmente se destinaron fondos para la expansión de la cobertura de la atención primaria. En particular, se crearon los Servicios de Atención Primaria de Urgencia (SAPU) y se implementaron los terceros turnos. Sin embargo, a pesar de los esfuerzos financieros destinados a mejorar la situación del sector de la salud, la percepción del público sobre la gestión del gobierno en el área fue deteriorándose progresivamente. La discordancia entre el esfuerzo del gobierno y los resultados que se obtenían en las encuestas motivó un intenso debate en el interior de los equipos de gobierno. La controversia creció a propósito de la elaboración de la plataforma electoral del segundo Gobierno de la Concertación. Mientras algunos sostenían que el gasto aún no había aumentado lo suficiente y que la estrategia debía ser continuar con más de lo mismo, otro sector que también había participado de las tareas de gobierno puso el énfasis en la deficiente gestión y en la falta de orientación a la gente como las fallas fundamentales de la estrategia seguida. Para este último sector, más allá de situaciones coyunturales o transitorias que pudieran explicar por qué el incremento de los recursos no se tradujo en incrementos de la cantidad y calidad de la atención de salud, los resultados obtenidos eran consecuencia de un sistema que carecía de incentivos y reglas que permitieran una buena gestión. Así por ejemplo, la separación de funciones, principio básico para identificar responsables, no había logrado consolidarse. El mantenimiento de presupuestos históricos, la falta de competencia y la inexistencia de derechos explícitos de los beneficiarios permitían el predominio de la tecnocracia sobre los intereses de las personas.

\section{El segundo Gobierno de la Concertación: la búsqueda de una estrategia para aumentar la satisfacción de los usuarios}

El segundo Gobierno de la Concertación introdujo en su discurso los temas de mejorar la eficiencia y acercar la salud a la gente. En esta dirección, la política del gobierno se propuso mejorar la equidad, profundizar la aportes de los hogares, situación que tiene el potencial de imponer barreras adicionales a las ya existentes para el acceso a los servicios de salud. Resulta interesante notar que, en 1994, el sector público financiaba $76 \%$ del GNS en los países industrializados, mientras que en las regiones con menos industrialización o de menores ingresos la participación del financiamiento público estaba en torno a $50 \%$ o menos (cuadro 1), planteando así un desafío al logro del desarrollo humano.

El análisis de la composición pública-privada del GNS en la Región para el lustro de 1990 a 1995 muestra que la participación del gasto público disminuyó y la del gasto privado aumentó. El componente del gasto público atribuible a la seguridad social se redujo en 6,6 puntos porcentuales, mientras que los componentes atribuibles al gasto local y central aumentaron ligeramente (cuadro 2). Es de notar que dos terceras partes del gasto privado se realizaron por la vía de desembolsos de los hogares en la compra de servicios de salud.

Como ejemplo, la estructura del GNS en Argentina en 1997 muestra el patrón al que las reformas están conduciendo al sector de la salud. En primer lugar, se tiene una composición pública-privada de 43 y 57\%, respectivamente. En segundo lugar, se observa que, dentro del gasto público, el gasto del gobierno central solo representa $23 \%$ y que el resto corresponde a las provincias y a los municipios. Por último, se debe hacer notar la participación creciente de los gastos directos de bolsillo para pagar insumos y servicios que no cubren los planes de salud. ${ }^{4}$

La figura 3 muestra el gasto privado en salud en la Región como porcentaje del gasto total. Por un lado están Guyana y Costa Rica, con una baja participación del sector privado, y, por otro lado, la República Dominicana y Guatemala, con una alta participación del gasto privado. En la Región de las

4 Estimaciones propias a partir de la base de dato de Cuentas Nacionales del Programa de Políticas Públicas, División de Salud y Desarrollo, OPS/ OMS. 
CUADRO 1. Gasto en salud por región y nivel de ingreso, 1994

\begin{tabular}{lccc}
\hline \multicolumn{1}{c}{ Regiones } & Per cápita & \% PIB & $\begin{array}{c}\text { Gasto público como } \\
\% \text { del gasto total }\end{array}$ \\
\hline Países industrializados $^{\mathrm{c}}$ & 1777 & 8,3 & 76 \\
Países no industrializados: & & & \\
$\quad$ América Latina y el Caribe & 367 & 6,1 & $42^{\mathrm{d}}$ \\
Europa y Asia Central & 346 & 4,1 & 72 \\
Asia Oriental y Pacífico & 158 & 7,2 & 52 \\
África del Norte y Medio Oriente & 353 & 5,2 & 50 \\
Sudeste Asiático & 65 & 3,7 & 39 \\
África Subsahariana & 111 & 4,0 & 54
\end{tabular}

Fuente: Citado en Schieber G, Maeda A. Health care financing and delivery in developing countries. Health Aff 1999; 18:193-205. Tabla 4.

a En dólares con el ajuste cambiario de la paridad del poder adquisitivo.

b PIB: producto interno bruto.

c Países de la Organización para la Cooperación y el Desarrollo Económico (OCDE) con la exclusión de Hungría, México y Turquía.

d Estimación del programa de políticas públicas de la OPS/OMS.

CUADRO 2. Composición del gasto total (público y privado) en salud según subsectores como porcentaje del gasto total. América Latina y el Caribe, 1990 y 1995

\begin{tabular}{lcr}
\hline & \multicolumn{2}{c}{ Gasto en salud (\%) } \\
\cline { 2 - 3 } \multicolumn{1}{c}{ Subsector } & 1990 & 1995 \\
\hline Público & 43,4 & 41,5 \\
Gobierno Central & 12,2 & 15,8 \\
Gobierno Local & 7,4 & 8,5 \\
Seguridad Social & 23,8 & 17,2 \\
Privado & 56,6 & 59,5 \\
Directo & NDa & 39,0 \\
Indirecto & ND & 19,5 \\
Total & 100,0 & 100,0 \\
\hline
\end{tabular}

Fuentes: Para 1990: Suárez R et al. Gasto en salud y financiamiento. América Latina y el Caribe. Desafíos para la década de los noventa. OPS-Programa de Políticas Públicas (documento mimeografiado), 1995. Para 1995: OPS/ OMS. La salud en las Américas 1998. Washington, D.C.: OPS/OMS; 1998. Cuadro 54

a ND: no disponible.
Américas, 13 países cuentan con una participación mayoritaria del gasto privado y en otros 23 países predomina el gasto ejercido a través de instituciones públicas prestadoras de servicios.

El gasto directo de los hogares se orienta principalmente a cubrir las consultas médicas $(38,1 \%)$ y los medicamentos $(22,3 \%)$. En el caso de estos últimos, la mayor parte del gasto se realiza en medicamentos de marca y alejados del marco terapéutico que ha propuesto la OMS con base en los medicamentos esenciales. La extensión de los mecanismos de mercado en el funcionamiento del sector en la Región sin duda significará una mayor presión al aumento de los gastos, pues los precios de los bienes y servicios aumentarán.

Los estudios de cuentas nacionales que se han llevado a cabo en la Región bajo el proyecto "Reforma del Sector de la Salud en América Latina y el Caribe", en colaboración de la OPS,
USAID y otros socios, han permitido obtener información adicional sobre algunas características del GNS. Para los países participantes en este proyecto, se observa que el gasto se sigue orientando a la atención curativa $(72,6 \%)$, con solo $7 \%$ para la atención preventiva, $12,1 \%$ para gastos administrativos $y$ $8,3 \%$ para otros rubros, como infraestructura, compra de insumos y formación de recursos humanos (cuadro 3). La información proveniente de este tipo de estudios permitirá mejorar el proceso de monitorización y evaluación de los recursos financieros invertidos en el sector de la salud y hacer un uso de estos recursos con una mejor relación costo-efectividad.

\section{FINANCIAMIENTO: REFORMAS Y TENDENCIAS EN LA REGIÓN}

Las reformas del financiamiento del sector de la salud se iniciaron durante los años ochenta como respuesta a la necesidad de compensar la reducción del aporte fiscal y tuvieron como principal objetivo aumentar los recursos financieros; posteriormente se han revisado a la luz de los objetivos de la reforma del sector de la salud y de los programas económicos que buscan crecimiento con equidad.

En los países industrializados el financiamiento ha sido mayoritariamente público, en tanto que en la Región de las Américas el financiamiento proviene principalmente de los hogares (8). Mientras que en los países industrializados, incluidos los Estados Unidos, la participación del financiamiento público (a través de impuestos y cuotas de seguridad social) en el sector está creciendo, en la Región de las Américas se percibe una tendencia opuesta, al mismo tiempo que se empiezan a incentivar los seguros privados y el pago por parte de los hogares (9).

La OPS propone un esquema de análisis y diseño de la política de financiamiento del sector de la salud que parte del reconocimiento de que este debe incluir simultáneamente el componente de atención a las personas y el conjunto de intervenciones de salud pública. Entre estas últimas se 
FIGURA 3. Gasto privado en salud como porcentaje del gasto total en salud (GNS), 1997-1998

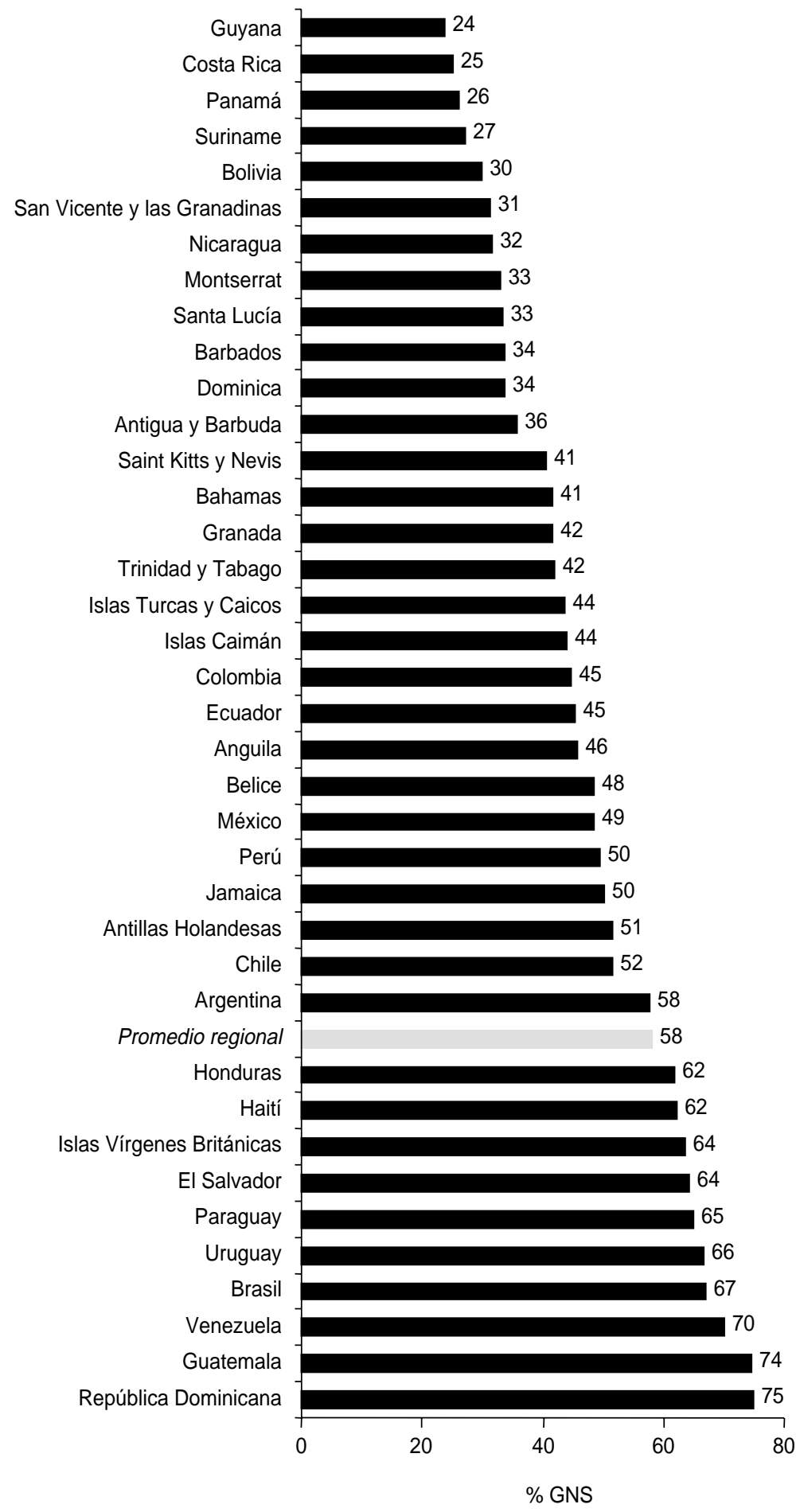

incluyen tanto las que tienen características de bienes públicos como las que, teniendo características de bienes privados, presentan alta externalidad positiva (por ejemplo, los programas de vacunación).
Tradicionalmente, se han identificado cuatro mecanismos de financiamiento que son: el gasto público a través de la recolección de impuestos generales o específicos para el financiamiento de la salud, los seguros pri- vados de salud, los seguros nacionales de salud y el cobro a los usuarios. $\mathrm{Si}$ bien estos cuatro mecanismos tienen un impacto positivo en el logro del objetivo de generar ingresos, también pueden tener un impacto negativo en el 
acceso a los servicios de salud, en la medida en que se transformen en barreras financieras a la expresión de la demanda de atención de salud por parte de las familias. Cada uno de ellos presenta ventajas y desventajas para el logro de los objetivos de sostenibilidad financiera, accesibilidad, equidad y eficiencia. El caso típico lo constituye el cobro a los usuarios, que, aunque tiene el potencial de generar ingresos financieros, también funciona como barrera al acceso y como causante de un financiamiento regresivo. En este sentido, se plantea un dilema en la selección del conjunto de mecanismos más adecuados para conformar una estrategia de financiamiento de la salud que permita lograr, simultáneamente, los objetivos múltiples de los procesos de reforma.

Con base en un análisis del potencial que cada uno de los mecanismos de financiamiento tiene para facilitar o limitar el logro de los objetivos de los procesos de reforma, la OPS propone la utilización conjunta de los mecanismos tradicionales con otros complementarios, en forma de subsidios, cuyo propósito es compensar los efectos no deseados de los mecanismos tradicionales y proteger el acceso de la población que se considere vulnerable por razones de nivel de ingreso o por condiciones específicas de salud. La propuesta considera el financiamiento público como el más eficiente y equitativo para las intervenciones de salud pública y sugiere que el debate que los países tengan en la definición de su estrategia de financiamiento se limite a los servicios de salud de atención a las personas (10).

La composición del financiamiento en la Región es heterogénea. En los ocho países que cuentan con estimaciones de Cuentas Nacionales de Salud se puede observar el peso mayoritario que tiene el financiamiento de los hogares en el sector. El caso extremo lo constituye la República Dominicana, donde $75,1 \%$ del financiamiento proviene de los hogares y el financiamiento público solo representa 14,4\% (cuadro 4).

La expansión de los mecanismos del mercado en los servicios de salud de la descentralización y estimular la participación social. La descentralización adquirió una doble dimensión: geográfica y de separación de funciones. Acercar la salud a la gente requería cambiar los sistemas de asignación de recursos y constituir una estructura organizacional que pudiera dirigir este proceso, que requería también que los sistemas de contratación e incentivos a los trabajadores fuesen adecuados. En este contexto, las principales medidas implementadas fueron:

Generar una mayor separación de funciones dentro del sistema público (hacia la creación del seguro público de salud)

En primer lugar, se redefinió la misión del FONASA, que asumió un nuevo papel en términos de administrador del seguro público colectivo de salud. En este contexto, el FONASA debería encargarse de la recaudación de fondos (cotizaciones, aporte fiscal y copagos), para con ellos comprar luego las prestaciones a los proveedores de salud. Esta modificación requirió que el ministerio cediera al FONASA las funciones de asignador de recursos a los establecimientos del SNSS. Esta primera fase de la RS se hizo en el contexto de la legislación vigente, que definía al FONASA como el responsable de la función de financiamiento, y operó con las restricciones propias de un cuerpo legal que no fue hecho de manera específica con ese propósito. Solo en 1997 el Gobierno anunció su decisión de introducir las reformas legales para consolidar la labor del FONASA como un efectivo comprador y administrador de las cotizaciones. A finales de 1999 se aprobó la correspondiente ley.

A pesar de las dificultades jurídicas, entre 1994 y 1996 el FONASA logró desarrollar los mecanismos de gestión necesarios para controlar sus ingresos e implementó un nuevo sistema de pago destinado a mejorar la eficiencia de los prestadores. La política de gestionar para sus beneficiarios le permitió mejorar fuertemente en la percepción de la gente. Los resultados de encuestas realizadas durante este período muestran con claridad una mejora en la percepción de las ventajas del seguro público.

\section{Modificar los mecanismos de asignación de recursos a los prestadores públicos}

Durante este período se comienzan a cuestionar los mecanismos de asignación de recursos existentes para transferir fondos desde el nivel central hacia los establecimientos públicos de salud. La combinación de transferencias discrecionales con base histórica, que representaban alrededor de 70\% del total, y la facturación por atención prestada (FAP) no habían generado incentivos para la eficiencia. La mayor parte de los recursos asignados por transferencias históricas respaldaban el gasto en recursos humanos y no estaban asociados al cumplimiento de ningún tipo de metas de gestión. Por su parte, el pago por atenciones prestadas en el que se basa el sistema FAP no incentiva la resolución de problemas de salud, sino que, por el contrario, reciben más aquellos prestadores que realizan más prestaciones para la solución de un mismo problema de salud. Un ejemplo de esta situación lo constituye el hecho de que el 
hospital que tiene a sus pacientes un mayor número de días hospitalizados, para una misma intervención quirúrgica, recibe más recursos.

En la atención secundaria y terciaria se estableció el sistema de pago asociado a diagnóstico (PAD) para un grupo de 26 prestaciones de mayor frecuencia, combinado con un sistema de pago prospectivo por prestación. Este sistema se inició en 13 de los 26 Servicios de Salud. El sistema PAD aplicado en los hospitales consiste en definir estándares para las prestaciones quirúrgicas más frecuentes, lo cual permite premiar a los establecimientos que son más eficientes en la solución de un problema. La elección de un número reducido de prestaciones, solo las más frecuentes, para la aplicación del sistema se justificó en buena parte por los costos administrativos del mismo. En el resto de las prestaciones, el control del costo se ejerce por la vía de un contrato de tipo prospectivo que establece de manera global las cantidades máximas de atenciones a ser financiadas por el FONASA.

La filosofía de estos métodos de pago persigue generar un marco de relaciones con los proveedores de salud que incentive la utilización de métodos eficientes, a la vez que intenta aumentar la transparencia en la asignación de los recursos, eliminando paulatinamente las transferencias de carácter histórico, no asociadas a la productividad ni a los resultados.

En la atención primaria municipalizada se estableció un sistema de pago per cápita que financia dicha atención según la población inscrita en cada comuna y diferencia estos pagos según el nivel de pobreza y el carácter urbano o rural de las comunas. De esta manera, se busca hacer el sistema más equitativo, ya que las comunas rurales y las más pobres reciben un pago per cápita más elevado. Como elementos para promover la eficiencia se utilizan la libertad de inscripción y los compromisos de gestión.

El sistema de pago per cápita estimula a los prestadores a preocuparse preferentemente por acciones de carácter preventivo. Adicionalmente, busca promover la competencia entre los establecimientos por la vía de permitir la elección del consultorio.

\section{Introducir los compromisos de gestión como herramienta} para incrementar gradualmente la eficiencia de los prestadores

Para fortalecer el proceso de descentralización y mejorar la eficiencia de la gestión, a partir de 1995 se establecieron compromisos de gestión entre el nivel central y los Servicios de Salud. El acuerdo, de carácter anual, establece el tipo de servicios a brindar, el nivel de asignación y transferencia de recursos, las metas de crecimiento y los indicadores de desempeño para evaluar las metas acordadas, que son fundamentalmente índices de actividad. Desde el punto de vista del cambio en los mecanismos de asignación de recursos, el compromiso de gestión cumple dos funciones importantes: permite operacionalizar el contrato prospectivo de recursos y permite establecer la gradualidad necesaria en la asignación de recursos. A modo de ejemplo, si un Servicio de Salud recibía antes de la reforma una cantidad de recursos superior a su producción, el compromiso de gestión permite mantener la transferencia por un período, sujeto al cumplimiento de un programa de ajuste que puede contemplar expansiones de producción y/o reducciones de costos.
Región ha hecho que una de las modalidades de financiamiento que se extendiera fuera la del cobro a los usuarios. En principio, el objetivo de este mecanismo consiste en focalizar el gasto público, cobrar por los servicios a las familias que tienen capacidad de pago y eximir o cobrar tarifas menores a las que no la tienen. La experiencia demuestra que esta modalidad de financiamiento se ha transformado en una barrera para el acceso a los servicios de salud y que su implementación ha generado problemas adicionales.

En primer lugar, resulta difícil sostener un sistema de tarifas diferenciadas cuando las excepciones han de hacerse caso por caso, en oposición a un esquema general de identificación de la población beneficiaria del gasto público. En muchas ocasiones, los establecimientos de salud tuvieron que reasignar tiempo del personal entrenado para atender a los pacientes a la tarea de identificar la capacidad de pago de los usuarios. Esto agregó ineficiencia al sistema, ya que estos profesionales tuvieron que reducir el tiempo dedicado a la atención de los pacientes.

En segundo lugar, cuando a los establecimientos se les permitió retener la totalidad o parte de la recaudación por cobro a los usuarios, la política de cobrar una tarifa preferencial a la población pobre o perteneciente a un determinado grupo identificado como vulnerable se transformó en un desincentivo al proveedor para atender a estas personas, debido a que no se estableció un mecanismo de compensación por los ingresos que los proveedores dejaron de recaudar a causa de la tarifa preferencial. En los casos en que no se permitió mantener la recaudación en el establecimiento, o en algún nivel intermedio o central en el Ministerio de Salud, el resultado final fue que los establecimientos públicos de salud terminaron ejecutando una actividad para la cual no tenían ni mandato ni ventaja comparativa, ya que la función de recaudación fiscal es ejecutada mejor por los servicios de impuestos.

La participación del financiamiento privado está correlacionada con los niveles de inequidad en la carga financiera de los hogares. Por ejemplo, en 
CUADRO 3. Distribución del gasto en salud por tipo de servicios en ocho países de la Región, 1995

\begin{tabular}{lcccc}
\hline \multicolumn{1}{c}{ País } & $\begin{array}{c}\text { Atención } \\
\text { preventiva } \\
(\%)\end{array}$ & $\begin{array}{c}\text { Atención } \\
\text { curativa } \\
(\%)\end{array}$ & $\begin{array}{c}\text { Administración } \\
(\%)\end{array}$ & $\begin{array}{c}\text { Otros rubros } \\
(\%)\end{array}$ \\
\hline Bolivia & 2,8 & 61,8 & 10,4 & 25,0 \\
Ecuador & 2,3 & 83,4 & 5,5 & 8,8 \\
El Salvador & 7,8 & 71,4 & 8,2 & 12,6 \\
Guatemala & 4,0 & 77,6 & 10,5 & 7,9 \\
México & 9,0 & 80,0 & 3,0 & 8,0 \\
Nicaragua & 17,5 & 75,6 & 6,3 & 0,6 \\
Perú & 5,6 & 64,6 & 6,8 & 23,0 \\
República Dominicana & 7,4 & 67,0 & 15,5 & 10,1 \\
$\quad$ Promedio & 7,0 & 72,6 & 8,3 & 12,1 \\
\hline
\end{tabular}

Fuente: Estudios de cuentas nacionales. Colaboración de Organización Panamericana de la Salud/United States Agency for International Development/Partnerships for Health Reform.

CUADRO 4. Participación de las principales fuentes en el financiamiento de la salud en ocho países de la Región, 1995

\begin{tabular}{lcccc}
\hline \multicolumn{1}{c}{ País } & $\begin{array}{c}\text { Gobierno } \\
(\%)\end{array}$ & $\begin{array}{c}\text { Empresas } \\
(\%)\end{array}$ & $\begin{array}{c}\text { Hogares } \\
(\%)\end{array}$ & $\begin{array}{c}\text { Externas } \\
(\%)\end{array}$ \\
\hline Bolivia & 18,4 & 38,9 & 32,5 & 10,2 \\
Ecuador & 38,7 & 3,7 & 48,6 & 9,0 \\
El Salvador & 35,8 & 9,5 & 50,2 & 4,5 \\
Guatemala & 31,9 & 19,2 & 43,0 & 5,9 \\
México & 14,4 & 21,8 & 63,6 & 0,2 \\
Nicaragua & 42,0 & 12,0 & 23,0 & 23,0 \\
Perú & 34,0 & 28,0 & 37,0 & 1,0 \\
República Dominicana & 14,4 & 8,7 & 75,1 & 1,8 \\
$\quad$ Promedio & 28,7 & 26,1 & 38,2 & 7,0 \\
\hline
\end{tabular}

Fuente: Estudios de cuentas nacionales. Colaboración de Organización Panamericana de la Salud/United States Agency for International Development/Partnerships for Health Reform.

Honduras, país donde el sector privado contribuye con $62 \%$ del financiamiento, el cuartil de la población con menores ingresos destina $7,1 \%$ de sus ingresos familiares al gasto en servicios y bienes de salud, mientras que el cuartil de mayores ingresos destina solo $2,5 \%$ a este fin. Por el contrario, en Costa Rica, donde la participación del financiamiento privado es menor $(25 \%)$, se observa una mayor progresividad, ya que el cuartil de menores ingresos destina $2,2 \%$ a la salud y que este porcentaje aumenta hasta llegar a $4,5 \%$ en el cuartil más rico. En estas cir- cunstancias, al Estado le corresponde un importante papel como ente rector para corregir las fallas del mercado que limitan el acceso a los servicios de salud (11).

\section{CONCLUSIONES}

Las modificaciones del financiamiento del sector de la salud han sido un componente sustancial de los procesos de reforma del sector en las Américas. El objetivo explícito ha pasado de uno que buscaba exclusiva- mente la sostenibilidad financiera a uno que busca, simultáneamente, el logro del acceso equitativo a servicios de calidad.

El estudio del financiamiento y del gasto en salud debe estar siempre presente en los estudios sobre reforma, ya que las modificaciones en este área repercuten en el logro de los objetivos de accesibilidad, equidad, efectividad y eficiencia. La información económica puede facilitar una evaluación integral del funcionamiento del sector.

El análisis de los resultados de salud en la Región indica que son relativa- 
Orientar los nuevos recursos hacia programas que incrementan las atenciones

Como elemento central de la nueva estrategia, los nuevos recursos se destinaron a incrementar las atenciones de salud. Se pusieron en marcha programas de expansión en las áreas de atenciones complejas con mejor relación costo-efectividad, de atención del adulto mayor y de reducción de listas de espera quirúrgicas. Producto de esta experiencia, en 1997 el FONASA incorporó en los Compromisos de Gestión, los llamados compromisos de oportunidad en la atención del usuario. Como culminación de esta política, durante 1999 el programa de enfermedades de alto costo evolucionó a un seguro de riesgos catastróficos.

\section{Cambiar el sistema de incentivos al personal}

Para tratar de que las formas de contratación y sistemas de remuneración incentiven la expansión de la atención y la mejora en la calidad, se negoció con el Colegio Médico la reforma del estatuto jurídico con el fin de "crear un marco jurídico que establezca un nuevo sistema de relaciones laborales y de remuneraciones para los principales gremios médicos que se desempeñen en los establecimientos asistenciales pertenecientes al SNSS." Dicho proyecto de ley contempla la creación de bonificaciones al desempeño y un conjunto de asignaciones que le permiten al sistema público tener mayor competitividad frente al sector privado. Después de casi cinco años, el proyecto aún no ha sido aprobado en el Parlamento. En 1996, tras su aprobación en primer trámite, el gobierno lo retiró para renegociarlo con el gremio médico. De allí en adelante no hubo motivación para acelerar su trámite.

\section{Los problemas del proceso}

El proceso de transformación no ha estado exento de problemas. En lo sustancial, las resistencias han venido de los prestadores públicos y municipales y se han manifestado en conflictos declarados por parte de los gremios en algunos hospitales y consultorios municipales. La solución de dichos conflictos ha pasado por postergar las medidas de ajuste que se debían tomar, lo que ha aplazado la plena vigencia de la RS. Estas situaciones han puesto el proceso de RS en una situación compleja, ya que la credibilidad de los cambios anunciados se ha visto seriamente afectada. Por su parte, los cambios legales necesarios para consolidar y avanzar en el proceso de separación de funciones se han visto obstaculizados por el conflicto de objetivos que suele darse en los procesos de descentralización entre la visión de los responsables de las finanzas públicas y los encargados de la RS.

En resumen, a partir de 1994 se inició un proceso de reforma destinado a introducir mayor eficiencia y orientar el sistema público hacia el usuario. Aunque ha tenido éxitos importantes, no está aún consolidado. mente pobres en función del volumen de recursos que se les destina. Esto sugiere la necesidad de evaluar el destino del gasto desde el punto de vista de su eficiencia tanto técnica como asignativa. Es indudable que se tiene que mejorar la información financiera del sector para poder evaluar mejor su desempeño.

La búsqueda simultánea de varios objetivos plantea el reto de considerar el financiamiento del sector desde una perspectiva de estrategia que identifique la mejor combinación de mecanismos alternativos. La OPS propone una combinación de esquemas tradicionales de generación de ingresos (gasto público vía impuestos, seguros privados de salud, seguros nacionales de salud y cobro a los usuarios) con esquemas complementarios de subsidios destinados a compensar a los grupos vulnerables de los efectos de las barreras financieras que reducen su demanda de atención de salud.

Como consecuencia de la aplicación de algunos mecanismos de mercado, los hogares aumentan su aporte al financiamiento del sector. Generalmente, este aporte ha sido regresivo, ya que los grupos de menores ingresos destinan un porcentaje mayor de los mismos a la obtención de atención de salud.

Para los países de la Región de las Américas queda claro que, debido a los niveles de pobreza y a las características de la distribución de los ingresos, los objetivos de ampliación de la cobertura y equidad en el acceso no se pueden lograr a través de un financiamiento que se sustente únicamente en los mecanismos de mercado, en los cuales la capacidad de pago depende principalmente del nivel de ingresos familiares.

Teniendo presente que las modificaciones de los modelos económicos que siguen los países de la Región han pasado por la redefinición del papel del Estado, destacando su función de garante del acceso a los servicios sociales, se plantea la necesidad de fortalecer el papel del Estado como corrector de las fallas del mercado que se presentan en el sector de la salud. 


\section{REFERENCIAS}

1. Banco Mundial. Informe sobre el desarrollo mundial. Washington, D.C.: Oxford University Press; 1993.

2. Organización Panamericana de la Salud. Las condiciones de salud de las Américas. Vol I. Washington, D.C.: OPS; 1994.

3. Govindaraj R, Chellaraj G, Murray CJ. Health expenditures in Latin America and the Caribbean. Soc Sci Med 1997;44:157-169.

4. Organización Panamericana de la Salud. La salud en las Américas. Washington, D.C.: OPS; 1998

5. Organización Mundial de la Salud. Informe mundial de la salud. Ginebra: OMS; 2000.
6. Organización Panamericana de la Salud. Cuentas nacionales de salud. Washington, D.C.: OPS; 1999.

7. Schieber G, Maeda A. Health care financing and delivery in developing countries. Health Aff 1999;18:193-205.

8. Anderson GF, Poullier JP. Health spending, access, and outcomes: trends in industrialized countries. Health Aff 1999;18:178-192.

9. Hsiao WC. A framework for assessing health financing strategies and the role of health insurance. En: Dunlop DW, Martins JM, eds. An international assessment of health care financ- ing. Lessons for developing countries. Washington, D.C.: World Bank; 1995. pp. 15-29.

10. Pan American Health Organization/United Nations Development Programme/Caribbean Community. Managing and financing health to reduce the impact of poverty in the Caribbean. Implementing decentralization and financing strategies while protecting the poor. Washington, D.C.: PAHO/UNDP/ CARICOM; 1999. (Policy Document)

11. World Bank. Sector strategy: health, nutrition, and population. Washington, D.C. World Bank; 1997.

ABSTRACT Being knowledgeable about national health expenditures and sources of financing is essential for decision-making. This awareness also makes it possible to evaluate the equity of allocation and the efficiency of utilization of these resources. Changes in fi-

Health spending and financing: the current situation and trends nancing have been a substantial component of health sector reform in the Americas. The goal has shifted from merely one of financial sustainability to simultaneously seeking equitable access to quality services. In this article the Pan American Health Organization (PAHO) presents a proposal for analyzing and designing a policy on health financing. The aim of the policy is to identify the mix of financing mechanisms most likely to simultaneously produce financial sustainability, equity, access, and efficiency. The PAHO proposal combines traditional mechanisms for generating resources (public funds from taxes, as well as private health insurance, national health insurance, and user fees) with complementary subsidy mechanisms for vulnerable groups. Health financing strategies ought to explicitly consider the financing both of care for individuals and of health interventions for the general public good, for which public financing is the most equitable and efficient approach. 